DEMOGRAPHIC RESEARCH

VOLUME 37, ARTICLE 30, PAGES 929-956

PUBLISHED 6 OCTOBER 2017

http://www.demographic-research.org/Volumes/Vol37/30/

DOI: 10.4054/DemRes.2017.37.30

Research Article

Too early or too late: What have we learned from the 30-year two-child policy experiment in Yicheng, China?

Yu Qin

Fei Wang

(C) 2017 Yu Qin \& Fei Wang.

This open-access work is published under the terms of the Creative Commons Attribution NonCommercial License 2.0 Germany, which permits use, reproduction, and distribution in any medium for noncommercial purposes, provided the original author(s) and source are given credit.

See http://creativecommons.org/licenses/by-nc/2.0/de/ 


\section{Contents}

$\begin{array}{lll}1 & \text { Introduction } & 930\end{array}$

$2 \quad$ Policy background $\quad 932$

$3 \quad$ Conceptual framework 935

$4 \quad$ Data sources 937

$5 \quad$ Empirical strategy $\quad 938$

$6 \quad$ Results 942

$\begin{array}{lll}7 & \text { Robustness checks } & 949\end{array}$

8 Conclusion 952

$9 \quad$ Acknowledgements 953

References $\quad 954$ 


\title{
Too early or too late: What have we learned from the 30-year two-child policy experiment in Yicheng, China? \\ Yu Qin ${ }^{1}$
}

Fei Wang ${ }^{2}$

\begin{abstract}

\section{BACKGROUND}

In January 2016, China ended its 35-year-old one-child policy and replaced it with a nationwide two-child policy. However, it remains unclear whether a two-child policy can effectively increase the fertility level in China.
\end{abstract}

\section{OBJECTIVE}

We reviewed the 30-year (1985-2015) two-child policy experiment carried out in Yicheng, a county in the Shanxi province of China, to assess the impact of this policy on the crude birth rate, as compared with the one-child policy implemented in most other places in Shanxi.

\section{METHODS}

We adopted a synthetic control approach. Using this method, we constructed a synthetic county using counties in Shanxi that were subject to the one-child policy. The synthetic county had similar observed characteristics to Yicheng before the launch of Yicheng's two-child policy experiment in 1985. Therefore, birth rate differences between Yicheng and the synthetic county after 1985 could be attributed only to the two-child policy.

\section{RESULTS}

We did not find any short-term impacts of the two-child policy on the Yicheng birth rate prior to the 1990s. We estimated that the two-child policy, in the long run, would lead to a maximum of two more births per 1,000 people every year in Yicheng, compared with similar areas that had a one-child policy.

\section{CONCLUSION}

The two-child policy was not found to boost the birth rate in Yicheng and similar places.

\footnotetext{
${ }^{1}$ Department of Real Estate, National University of Singapore. E-Mail: rstqyu@nus.edu.sg.

${ }^{2}$ Corresponding author; School of Labor and Human Resources, Renmin University of China, China.

E-Mail: fwang@ruc.edu.cn.
} 
Qin \& Wang: What have we learned from the 30-year two-child policy experiment in Yicheng, China?

\section{CONTRIBUTION}

The study identified the causal effect of a two-child policy, and was more methodologically reliable than related studies that primarily explored statistical correlations.

\section{Introduction}

To deal with the growing challenges of aging, an increasing number of countries have been adopting population policies designed to increase birth rates (United Nations 2013). The effects of such policies are generally mixed. However, they tend to fail more often in East Asia than in other regions (McDonald 2006). China, the most populous country in the world, began to follow a recent trend, and as of January 1, 2016, started to allow married couples to have two children, ending a 35-year-long one-child policy (OCP). Assessing the potential effects of China's policy on the change in fertility will not only add to the body of pronatal policy literature, but will also have implications for future policy adjustments in China.

It is still too early to evaluate the new policy directly. However, its effects can be estimated by investigating people's desire to have a second child, or by exploring the impact of two-child policies that have been previously implemented in China. Evidence based on the desire for a second child was neither consistent nor credible. ${ }^{3}$ Wang, $\mathrm{Hu}$, and Zhang (2013) simulated a scenario in which a two-child policy was implemented in 2015 , and found that the total fertility rate for 2016 would, at most, increase by 0.5 , or approximately 6 million births. However, Zhai, Zhang, and Jin (2014) found that the total fertility rate would increase to 3.0 with the institution of a two-child policy, which would account for almost 20 million more births in the first year the new policy was implemented.

In this paper, we looked into the case of Yicheng county, which was the only jurisdiction with a persistent two-child policy since 1985. Yicheng, a typical agricultural county in Shanxi province (location shown in Figure 1), was granted exemption from the OCP in 1985. The locality was designated an experimental zone in which almost all couples were given the option to have two children. This unique experiment provided an excellent opportunity for scholars to investigate the potential consequences of a two-child policy by using historical data.

$\mathrm{Gu}$ and Wang (2009), Wei and Zhang (2014) and Wu (2014) have all compared the demographics in Yicheng, both before and after the experiment, and to other

\footnotetext{
${ }^{3}$ Desire to have a second child often fails to predict actual behaviors (e.g., Adsera 2006).
} 
counties in the same prefecture city. They all concluded that replacing the OCP with a two-child policy had little positive impact on fertility levels. However, it has been challenging to quantitatively estimate the impact of the two-child policy in Yicheng in an unbiased way. On the one hand, before and after comparisons of Yicheng's birth rate may not accurately reflect the true effects of the population policy given possible changes in other determinants to fertility rates. Likewise, it is difficult to carry out a traditional difference-in-differences (DID) analysis, which would account for the time trends of birth rates in the treated and control jurisdictions. There are two reasons for this. First, any inference based on a DID analysis is likely to be biased if the number of treatment units was small. Second, the control and treatment units analysed must have parallel growth trends associated with the outcome variables prior to the policy experiment. This is referred to as the 'parallel trend assumption' in the DID framework. However, our data indicates that the birth rates in Yicheng, and the control counties that were studied, had significantly different growth patterns prior to the experiment, which violates the assumption.

In this paper we use the synthetic control approach to reexamine Yicheng's twochild policy experiment, and more accurately estimate its impact on the locale's fertility rate. The synthetic control method was most suitable for comparative case studies in which only one, or a few, treated units exist. Positive weights were calculated and assigned to a number of control units from a donor pool of counties in the same province, such that the weighted averages of birth rates in the selected control counties best mirrored Yicheng's birth rate trend prior to the two-child policy. The weighted average of the birth rate determinants from the control counties also matched their counterparts in Yicheng prior to the policy's implementation. The formation of a synthetic control unit provided a policy counterfactual, and allowed us to evaluate the effectiveness of the two-child policy with more accuracy.

Comparing the crude birth rate in Yicheng to a 'synthetic Yicheng,' we found that from 1985-1990, the first six years during which the two-child policy was in effect, the birth rate in Yicheng was not significantly different from that in other counties and districts within the same province. This is likely because both the two-child policy in Yicheng and the OCP in other counties were weakly enforced during that time. Nevertheless, the impact of this policy became apparent in the long run, once the policies were strictly enforced. The 2000 and 2010 population censuses revealed CBR differences of 2.35 and 1.97 for Yicheng and synthetic Yicheng, respectively, indicating that the two-child policy would lead to a maximum of two more births per 1,000 people per year, compared with the OCP.

This paper is one of the first to present a counterfactual analysis of the potential impact of relaxing the OCP on birth rate by using a two-child policy experiment. This research is likely to become an important reference for further relaxation of the OCP 
nationwide. Our analysis suggests that replacing the OCP with a two-child policy may have little impact on crude birth rates in the short term, and would have a rather limited impact, if any, compared with prevailing estimates in the long term. Given that our estimate is likely to be in the upper bounds of the true impact, the relaxation of the OCP may have a very limited impact on the overall birth rate and fertility level in rural areas similar to Yicheng.

The rest of the paper is organized as follows: Section 2 provides the background for Yicheng's two-child policy experiment; Section 3 presents a conceptual framework through which to consider the potential impact of the OCP's relaxation; Section 4 describes the data used in this paper; Section 5 introduces the empirical strategy; Section 6 presents the main findings; Section 7 describes the robustness checks performed; and Section 8 concludes the paper.

\section{Policy background}

China began to implement its OCP in 1980. Under this policy, a married couple was generally allowed to have, at most, one child. ${ }^{4}$ However, the OCP was difficult to enforce nationwide, especially in rural areas where the policy threatened to significantly reduce the household labour force and decrease a family's capacity to support agricultural production. Families in rural areas were also more likely to express a desire for male children, and birth controls reduced a couple's chances of having a son. Given the realities of implementing the OCP in rural areas, Chinese authorities began to relax the OCP in the mid-1980s, gradually allowing rural married couples to have a second child if the first child was female, so long as the couple waited a sufficient amount of time between the two births (Yang 2004). ${ }^{5}$

Some scholars proposed alternative solutions to this caveat. In the spring of 1984 , Liang Zhongtang, a demographer in the Shanxi province People's Government Economic Research Center, proposed a restricted two-child policy that would require couples to marry later and wait longer before having a second child. In 1985, Liang's advocacy for this policy convinced the provincial government to allow Yicheng, a county in Linfen prefecture city, to replace the OCP with the two-child policy proposed by the demographer. Figure 1 shows the location of Shanxi province in China, and the location of Yicheng in Shanxi. Shanxi is in central North China, and Yicheng is located in South Shanxi.

\footnotetext{
${ }^{4}$ For additional details about the OCP and its earlier policy versions, as well as their effects on birth rate, see Wang (2014).

${ }^{5}$ Urban residents and rural residents whose first child was a male were generally subject to the one-child birth quota. Therefore, we still refer to the policy as the OCP.
} 


\section{Figure 1: Locations of Shanxi and Yicheng}
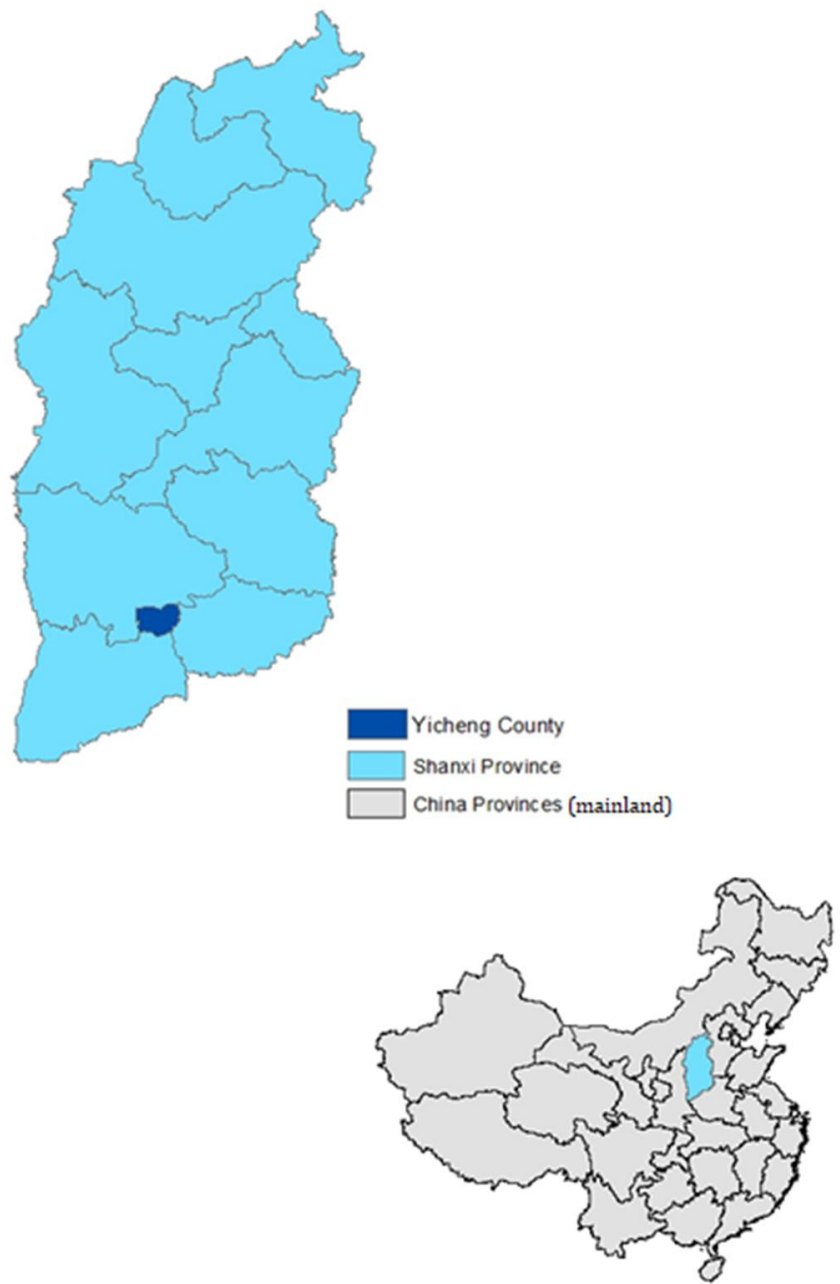

Note: The map is sourced from Gibbs Lab at Cornell University to visualize the geographic location of Yicheng in Shanxi and Shanxi in mainland China. This map does not represent the personal view of the authors on China's mainland territory. 
In fact, Yicheng was not chosen for this experiment at random. In an interview, ${ }^{6}$ Liang summarized the three reasons why Yicheng was selected as a pilot for this experiment. First, it was logical to test the two-child policy in a county with a primarily rural population given that it was more difficult to enforce the OCP in rural areas compared with cities. In 1985, over $90 \%$ of Yicheng's population lived in rural areas. Second, Yicheng had railroad access; therefore, program implementation and monitoring could be executed more easily. Lastly, the experiment was welcomed and supported throughout Yicheng. In our research, we put forth our best effort to control for the demographic and socioeconomic variables that are correlated with the placement of the program.

Yicheng's two-child policy includes the following measures: 1) All couples are encouraged to delay marriage, to postpone parenthood, and to have fewer children; 2) The 'one child per couple' norm is enthusiastically promoted; 3) Rural one-child families are offered financial incentives (i.e., financial rewards and preferential access to education and health services); and 4) State employees and urban couples are limited to one child only, except under special circumstances. Rural couples fulfilling the following requirements are allowed to have two children: a) They must marry three years later than the minimum marriageable age, as specified in The Law of Marriage (men at 22, and women at 20); b) The wife bears her first child at 24 and the second at $30^{7}$ (the birth spacing requirement was adjusted from six years to four years in 2007); c) The wife must apply for a birth permit for her second child and wait for a quota; and d) The couple must use effective contraception after their first child is born and must accept sterilization after their second child. Births beyond two children are strictly prohibited without exception and are subject to financial and disciplinary sanctions (Wei and Zhang 2014).

There are limited references related to the enforcement of Yicheng's two-child policy experiment. In the first decade of the experiment in Yicheng, more than half of the second children born were birthed without a permit because the parents violated the late marriage requirement or had not waited long enough between children to conceive. However, the ratio of unpermitted second children significantly dropped after 1995, and reached almost zero in 2010, as late marriages and larger gaps between pregnancies became more prevalent (Wu 2014).

Yicheng was the first county to implement the two-child policy experiment, but it was not the only one. A dozen more pilot counties, including one jurisdiction in Shanxi (i.e., Xinrong district in Datong prefecture) and a number of counties in other provinces also adopted this law. However, starting in the late 1980s, the two-child policy in most

\footnotetext{
${ }^{6}$ Jiao, H. (August 21, 2012,). Interview Liang Zhongtang, a Family Planning Expert and Demographer. Legal Weekly. Retrieved 5 September 2017, from http://www.legalweekly.cn/article_show.jsp?f_article_id=3804.

${ }^{7}$ Women who only want one child do not have to marry or give birth at an older age.
} 
of the pilot regions were replaced by the hybrid one-child policy, in which a rural couple could have two children only if the first child was female. Gu and Wang (2009) noted that the two-child policy was maintained in four regions: Chengde, in Hebei province; Enshi, in Hubei province; Jiuquan, in Gansu province; and Yicheng, in Shanxi province. Nevertheless, Liang (2014b) argued that Yicheng was the only appropriate location in which to study the two-child policy because Chengde's policy was only enforced in the least developed mountain areas. Because Enshi had a large ethnic minority population, the policy effects there may not have been representative; and Jiuquan's policy was often interrupted and remained illegal during most of the 1990s. Therefore, our research focused on the two-child policy in Yicheng.

\section{Conceptual framework}

This paper estimated the impact of a two-child policy on population growth compared with an OCP at the aggregate (county) level. The only measure of county fertility levels available to us was crude birth rate (CBR, i.e., the number of births per 1,000 per year). In contrast to total fertility rate as adjusted for age of the mother, the CBR of a county was likely to be affected by the gender and age structure of the county (Easterlin 1978). For example, more females of childbearing age in a county could lead to a higher CBR. Therefore, we needed to collect information on the gender and age distributions of selected counties for our analysis.

In addition to gender and age distribution, the choice of covariates relied on fertility theories. The readiness-willingness-ability (RWA) framework is a general tool for analysing fertility behaviours. Readiness indicates an economic benefit-cost analysis of behaviours. Willingness means normative acceptability of behaviours and is usually linked to cultural factors. Ability represents availability of new techniques (Lesthaeghe and Vanderhoeft 2001).

Easterlin and Crimmins (1985) proposed a supply-demand framework for fertility analysis, and the framework is, to large extent, consistent with the RWA framework. The supply-demand theory indicates that fertility levels are jointly determined by the demand for children, the supply of children, and the costs of fertility regulation. The demand for children corresponds to the economic benefit-cost analysis and is affected by household tastes, income, and the cost of raising children. The supply of children consists of natural fertility (e.g., fecundity), which may partly be influenced by cultural factors (Easterlin and Crimmins 1985), and the chances of child survival. The costs of

fertility regulation include people's attitudes about family planning, the accessibility of fertility control methods and supplies and, in the context of China, penalties for 
violating family planning policies. The costs of fertility regulation fall under the ability precondition of the RWA framework (Lesthaeghe and Vanderhoeft 2001).

We followed the supply-demand theory to select covariates, regarding the RWA framework as a supplement. On the demand side, we used county-level income indicators to capture the effects of income. The cost of raising children includes both direct costs, such as the cost of consumables like food and diapers, which was not available to us, and indirect costs, i.e., opportunity costs of raising children, such as parental wages, particularly mothers' wages. As county-level wages were unavailable, we considered county-level education variables as proxy measures (Zhang 1990). Education may also be associated with tastes in fertility, parental productivity of childbearing, and the incidence of child mortality (Zhang 1990), where the latter two factors belong to the supply side of the framework. Zhang (1990) also included parental residence, and we considered the proportion of the rural population accordingly. On the supply side, in addition to education, we considered age structure, income indicators, and health variables, which are related to nutritional and biological factors that govern fecundity (Frisch 1982; Wood 1989). Income and health factors may also be related to infant mortality rates.

We assumed that the differences in the costs of fertility regulation between Yicheng and other counties were accounted for in the principle differences in family planning policies. The policies also differed between urban and rural areas, and between ethnic majority Han communities and minority communities (Wang 2014). Therefore, the geographic and ethnic makeup of the population also needed to be considered so that the differences in policy intensity between Yicheng and the other counties studied were solely related to those counties' distinct policies, rather than the geographic and ethnic distribution of the population. Thus, in our analysis, we considered the share of the population that lives in rural communities and the ethnic distribution of the population by county. Unfortunately, county-level data on the ethnic makeup of the population was not available. Nevertheless, this is unlikely to be a problem in our study because Shanxi province was home to very few ethnic minorities in past decades. ${ }^{8}$

Culture is highlighted in the RWA framework and is an important determinant of fertility, both in China (Arnold and Liu 1986) and in other countries (Fernández and Fogli 2006). However, like direct costs of raising children, culture was unmeasurable in our study. To address the issue, we took advantage of a factor model (details introduced in Section 5) which includes such unobserved factors and allows their effects on fertility to change over time.

\footnotetext{
${ }^{8}$ The 1953 Chinese census showed that the percentage of ethnic minorities in Shanxi was only $0.14 \%$. By the 2010 census, the figure had risen slightly to $0.26 \%$. The percentages have been similarly low in almost all counties within Shanxi.
} 


\section{Data sources}

As stated above, the primary variables of interest are county-level CBR and its determinants in Shanxi province. We restricted our data to counties in Shanxi for two reasons. First, the number of counties in Shanxi was large enough to perform a comparative case study; and second, there would likely be more similarities between unobservable characteristics at the county level for counties within the same province.

We obtained CBRs and crude death rates (CDR, number of deaths per 1,000 people per year, used to measure health) for all 116 counties and districts in Shanxi from 1949 to 1990 (from the published book Forty years of population in Shanxi: 1949-1990). ${ }^{9}$ The data availability varied across jurisdictions. The shortest panel contained population measures from 1972 to 1990, while the longest recorded both variables continuously from 1949 to 1990 . We excluded Xinrong district in Datong prefecture from our sample because Xinrong was one of the localities that implemented a two-child policy during the late 1980s and early 1990s, which might have had an impact on the results. In addition, we adjusted our data to correct the number of administrative units analysed during our sample period. ${ }^{10}$ To assess the long-term impact of Yicheng's two-child policy, we also collected the CBR and CDR for all counties in Shanxi from the 2000 and 2010 population censuses. ${ }^{11}$

In addition to CDR, we required other predictors of CBR. Based on the literature discussed in the above section, we included the following sets of variables: 1) population by age cohort (age $0-14,15-59$, and 60 and above), and gender for each prefecture from the 1982 population census. Because age by gender data was not available at the county level, we assumed that the age-gender distribution in each county was the same as the prefecture it was affiliated with. ${ }^{12}$ In addition, we used population by gender data for each county from 1949-1990 to complement the prefecture-level age-gender data, as per Forty years of population in Shanxi: 1949-

\footnotetext{
${ }^{9}$ Another widely used measure of fertility is the total fertility rate (TFR). However, TFR data is not available at the county-year level.

${ }^{10}$ We removed Yanbei district from our sample as it was disbanded in 1993, and we reassigned the 10 affected counties to their corresponding prefectures based on the current administrative hierarchy. Specifically, we assigned Tianzhen, Yanggao, Guangling, Lingqiu, Hunyuan, Zuoyun, and Datong counties to Datong prefecture; and Huairen, Ying, and Youyu counties to Shuozhou prefecture.

${ }^{11}$ There were a large number of administrative changes in the 2000 and 2010 population censuses compared with earlier data, especially in the urban districts. If the administrative boundary did not change, we simply matched the new jurisdiction name with the old name. In addition, we made three further changes: 1) We matched the weighted average of Jinyuan district and Xiaodian district in Taiyuan prefecture to the old Nanjiao district in Taiyuan; 2) We matched the weighted average of urban and suburban districts in Changzhi prefecture to the old urban district in Changzhi; and 3) We matched the urban district and Zezhou county in Jincheng prefecture to the old urban district in Jincheng.

${ }^{12}$ The ten counties administered by Yanbei district, which was later disbanded, were assigned values based on Yanbei district.
} 
1990. These variables were used to control the variations in CBR that occurred as a result of the differences in the age-gender distribution. 2) Other indicators included the rural proportion of the population, GDP per capita, rural personal income, number of elementary schools per 1,000 people, number of middle schools per 1,000 people and number of hospitals per 1,000 people. For districts and counties in Linfen prefecture, with which Yicheng is affiliated, we collected data on these variables at the county level from Fifty years of Linfen, a publication that contains yearly statistics for all jurisdictions in Linfen prefecture. For other counties and districts, we were only able to obtain the rural proportion of the population at the county level from Forty years of population in Shanxi: 1949-1990. ${ }^{13,14}$ The rest of the variables were only available at the prefecture level and were obtained from Sixty years of brilliant Shanxi province. ${ }^{15}$ For these variables, we assumed they had the same value at the county level as the prefecture the county was affiliated with.

\section{Empirical strategy}

The goal of this paper was to evaluate the causal impact of Yicheng's two-child policy on birth rate. A key concept in policy evaluation was to find the appropriate 'counterfactual,' i.e., what would have occurred had the policy not been in place. Because we could not observe the 'counterfactual' of the treated unit, we needed to find a good comparison group similar to the treated one, but unaffected by the policy. Research designs for identifying appropriate comparison groups can be categorized into randomized controlled trials (RCTs) and quasi-experimental designs (QEDs), the latter of which includes regression controls, difference-in-differences, propensity score matching, regression discontinuity, and so on. ${ }^{16}$ In the context of the Yicheng experiment, we needed to rely on quasi-experimental approaches to identify a credible policy counterfactual for Yicheng.

However, it was not easy to identify a control unit similar to Yicheng yet unaffected by the policy. Figure 2 illustrates the CBR trends in Yicheng and 114 other

\footnotetext{
${ }^{13}$ The data for the urban districts in Taiyuan, Yangquan, and Datong was missing. We inferred these values by using the rural proportion of the population for the entire prefecture.

${ }^{14}$ The data for Gujiao city in Taiyuan was missing. We inferred these values based on the rural proportion of the population in Taiyuan prefecture and the other jurisdictions in Taiyuan.

${ }^{15}$ The data in the three books Forty years of population in Shanxi: 1949-1990, Sixty years of brilliant Shanxi province, and Fifty years of Linfen was all sourced from local statistical and public security authorities. As the three books are compiled statistics rather than academic publications we didn't include them in the reference list.

${ }^{16}$ An extensive literature review on policy impact evaluation is available at the UK Magenta Book (https://www.gov.uk/government/publications/the-magenta-book).
} 
districts and counties in Shanxi province. These data clearly suggest that the CBR in Yicheng varied greatly from the rest of the province. First, the CBR in Yicheng was significantly below the average CBR in other jurisdictions. In addition, the CBR growth differed in Yicheng compared with other jurisdictions. A formal test of the differences between Yicheng and other jurisdictions was carried out using a DID analysis. Using the data for 115 counties from 1972-1990, 2000, and 2010, we regressed CBR on county dummies, year dummies, and the interactions of Yicheng and year dummies. ${ }^{17}$ Figure 3 shows the $95 \%$ confidence band for the coefficients of the interactions, with 1984 as the baseline year. Almost all the coefficients were significantly negative before 1985, indicating a significantly larger variation in CBR between Yicheng and other counties during these years compared with 1984. In addition, any pair of coefficients in adjacent years prior to 1985 were significantly different from one another, which clearly suggests different growth patterns for Yicheng and the rest of the province. Therefore, the estimated impact of the pilot two-child policy would likely be biased if we had directly implemented a DID analysis. Moreover, Cameron and Miller (2015) indicated that a DID model is likely to be inconsistent if there are too few treatment groups compared with control groups, which was the case in our study. ${ }^{18}$

As difference-in-differences cannot appropriately identify the policy impact of the Yicheng experiment, we adopted a synthetic control approach that sufficiently addressed the shortcomings of a traditional DID analysis. The synthetic control method (Abadie and Gardeazabal 2003; Abadie, Diamond, and Hainmueller 2010, 2015) allowed us to construct an artificial control group that almost exactly mimicked the growth patterns of the treated unit prior to the policy experiment.

\footnotetext{
${ }^{17}$ The standard errors are clustered at the county level.

${ }^{18}$ Other quasi-experimental designs were not appropriate in this context either. For example, propensity score matching assumes selection on observables, and thus requires as many covariates as possible in predicting probabilities of being treated. In contrast to traditional matching, the synthetic control approach underscores period-by-period matching of the pretreatment outcome and demands less information from covariates. Our data contained a fairly long and complete panel of outcome and a limited number of covariates, which were available only in selected years. In that sense the matching approach may not be able to create qualified comparison units, and the synthetic control approach is a more appropriate choice. Moreover, since there was only one treatment unit in our study, using traditional matching methods could lead to inaccurate estimation and inference.
} 
Figure 2: Crude birth rate in Yicheng and other counties in Shanxi

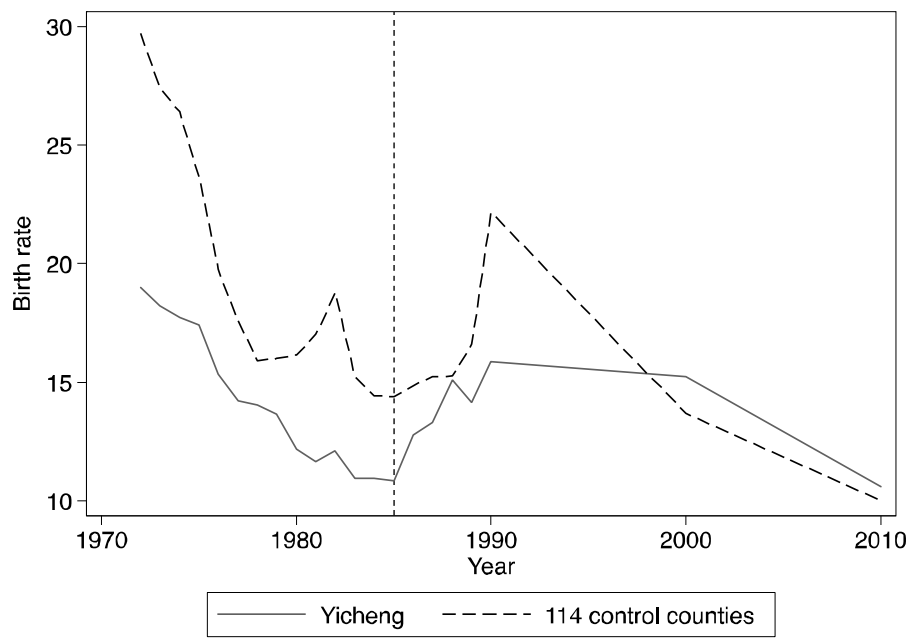

Data source: Please refer to Section 4.

\section{Figure 3: Difference-in-differences analysis}

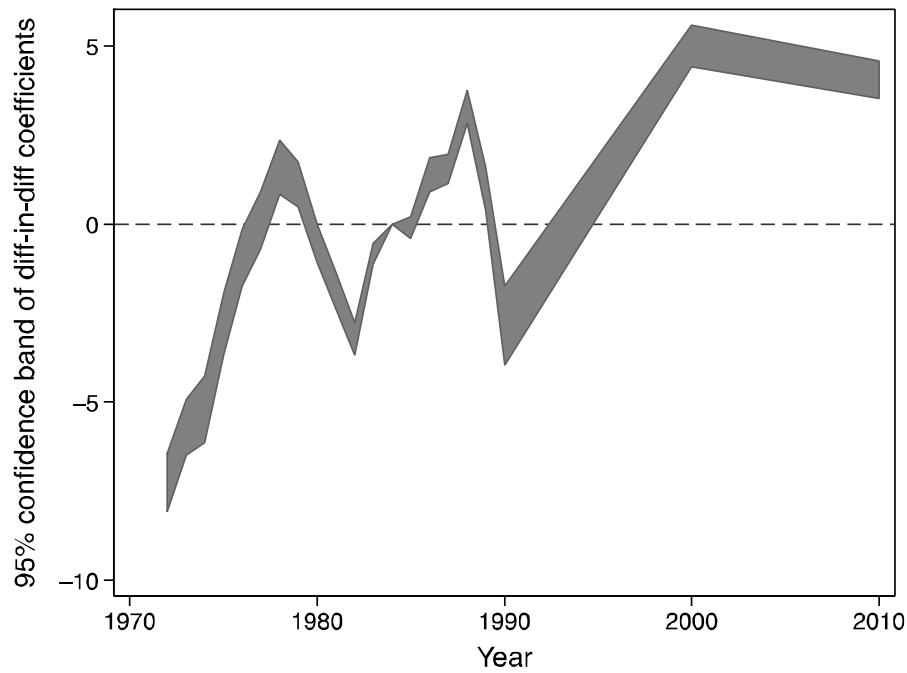

Note: Year 1984 is taken as the baseline year. 
Assume there are $J$ units. The first unit was the treated unit, and the remaining $J-1$ units were used to construct a synthetic control unit that was comparable to the treated unit. Assume $Y_{i t}$ is the dependent variable for unit $i$ over period $t . Y_{i t}$ was determined by the following factor model for $i=1,2 \ldots J$ in the pretreatment periods:

$$
Y_{i t}=\delta_{t}+\boldsymbol{\theta}_{t} \boldsymbol{Z}_{i}+\lambda_{t} \boldsymbol{\mu}_{i}+\varepsilon_{i t}
$$

In equation (1), $\delta_{t}$ is the period indicator. $\boldsymbol{Z}_{i}$ and $\boldsymbol{\mu}_{i}$ represent a set of observed and unobserved time-invariant characteristics of unit $i$, respectively; $\boldsymbol{\theta}_{t}$ and $\boldsymbol{\lambda}_{t}$ are coefficients of $\boldsymbol{Z}_{i}$ and $\boldsymbol{\mu}_{i}$. This factor model allowed time-varying effects for both observables and unobservables, which was less restrictive than traditional DID models with fixed effects. $\varepsilon_{i t}$ is the idiosyncratic error. Suppose there were a set of nonnegative weights $\left(w_{2}, w_{3}, \ldots, w_{J}\right)$ and $\sum_{j=2}^{J} w_{j}=1$, such that

$$
\begin{aligned}
& \sum_{j=2}^{J} w_{j} Y_{j t}=Y_{1 t}, \text { for each pretreatment period } \\
& \sum_{j=2}^{J} w_{j} \boldsymbol{Z}_{j}=\boldsymbol{Z}_{1}
\end{aligned}
$$

Given this, Abadie, Diamond, and Hainmueller (2010) showed that the treatment effect in any post-treatment period, $t$, would be $Y_{1 t}-\sum_{j=2}^{J} w_{j} Y_{j t}$, as long as the characteristics of the donor pool of units were not substantially different from those of the treated unit, and the pretreatment period used to match the treated unit to its synthetic counterpart was sufficiently long. ${ }^{19}$ Equations (2) and (3) are theoretical presentations. In practice, often no sets of weights could make the two equations hold exactly. Then, it was adequate to derive weights such that the equations hold approximately (Abadie, Diamond, and Hainmueller 2010). The weights were obtained by minimizing the distance between a vector of the treated unit, comprising the dependent variable in each year prior to the treatment, each independent variable in the pretreatment period, and a similar vector of the control units containing the weighted dependent variable in each year and each weighted independent variable in the pretreatment period from all units in the donor pool. ${ }^{20}$

\footnotetext{
${ }^{19}$ Abadie, Diamond, and Hainmueller (2010) also proved that, with such conditions, both observables and unobservables are matched between the treated unit and the synthetic control unit. In other words, selection biases from observed and unobserved factors will both be eliminated.

${ }^{20}$ More details are introduced in Abadie, Diamond, and Hainmueller (2010). They made a package of the synthetic control approach for various statistical software, called Synth, which was used to estimate the optimal weights in our paper. More information about Synth is available at https://web.stanford.edu/ $\sim$ jhain/synthpage.html.
} 
In our study, Yicheng is the treated unit, and the other 114 counties in Shanxi form the donor pool that was used to construct the synthetic Yicheng. Weights were assigned to the donor pool units such that 1) the CBR of Yicheng was as close as possible to the weighted average of the CBR for the donor pool units in each period prior to 1985 and 2) for each determinant of CBR, its average value over the preintervention periods in Yicheng was as close as possible to its counterpart in the weighted donor pool. Using this set of weights, a synthetic Yicheng was constructed from the donor pool, and mirrored Yicheng before the policy intervention in terms of CBR and its determinants.

Our core dependent variable was CBR at the county level. As introduced in previous sections, we included the following variables as predictors. First, we included variables related to gender by age cohort (ages $0-14,15-59$, and age 60 and above) population structure. Specifically, we included the share of males and females for the three age cohorts, respectively. It is also worth noting that the sex ratio under age 15 may reflect gender preferences in a given area to a certain extent. Second, we included health measures such as CDR and the number of hospitals per 1,000 people. Third, we considered a series of socioeconomic indicators including a) the rural proportion of the population, as CBR varied from urban to rural areas; b) GDP per capita and rural incomes in natural log form, which measured the income level of a region; and c) measures of the average educational level in each region, which likely affected CBR, and included the number of elementary schools and middle schools per 1,000 people. Lastly, Abadie, Diamond, and Hainmueller (2010) suggested that controlling for lagged values of the dependent variable helps to better fit the dependent variable's trend. Thus, we also included historical birth rates from 1972 to 1984 as predictors.

We used 1972-1984 as the sample period to fit the CBR growth trend between Yicheng and the synthetic Yicheng, as it is the longest period covered by all 115 counties and districts in Shanxi province. ${ }^{21}$ We did not include any jurisdictions outside Shanxi province because family planning policies varied from province to province, making the comparison less relevant. We then used the data from 1985 to 1990 to estimate the short-term impact, and the data from 2000 and 2010 to infer the long-term impact.

\section{Results}

As shown in Table 1, the synthetic control method generated positive weights for Kuangqu in Datong prefecture (0.302), Hexi district in Taiyuan prefecture (0.283), Fushan county in Linfen prefecture (0.195), Qinyuan county in Changzhi prefecture

\footnotetext{
${ }^{21}$ Except for Xinrong in Datong, which was affected by two-child policy before the early 1990s.
} 
(0.184), and Pianguan county in Xinzhou prefecture (0.036). The synthetic Yicheng was similar to Yicheng in many respects, as suggested in Table 2. Particularly, birth rates prior to 1985 appeared similar. The simple average birth rates of the entire donor pool were substantially larger than those in Yicheng, but the synthetic Yicheng values closely matched Yicheng values. However, three predictors were not balanced across the treated and synthetic control groups: mortality rate per 1,000 people, number of hospitals per 1,000 people, and the share of rural population. ${ }^{22}$ The synthetic Yicheng had a lower mortality rate, more hospitals, and a lower rural population. All these imbalances suggested that the synthetic control group was more urbanized, healthier, and thus likely to have a lower birth rate. Therefore, our method may have overestimated the impact of a two-child policy on birth rate.

Table 1: Weights of synthetic control groups

\begin{tabular}{ll}
\hline County & Weight \\
\hline Kuangqu, Datong & 0.302 \\
Hexi, Taiyuan & 0.283 \\
Fushan, Linfen & 0.195 \\
Qinyuan, Changzhi & 0.184 \\
Pianguan, Xinzhou & 0.036 \\
\hline
\end{tabular}

Note: The weights calculated by the authors are used to construct the synthetic control group.

Figure 4 presents the CBR in Yicheng and its synthetic counterpart over time. In general the synthetic control group closely followed the trend of birth rates in Yicheng prior to the implementation of the two-child policy in 1985. Both birth rate series showed downward trends before 1985, which reflected the effects of both family planning policies and socioeconomic development. The birth rate increased in both Yicheng and the synthetic Yicheng from 1985 to 1990, and then fell during 2000 and 2010. However, the synthetic control group's CBR appeared to fall at a faster rate than did the CBR of Yicheng.

\footnotetext{
${ }^{22}$ As birth rates in each pretreatment year were used as predictors, the synthetic control approach assigned lower weights to the covariates in the matching optimization. As a result, the matching quality of covariates was not so adequate as birth rates. We also used the average pretreatment values of birth rates, instead of its value in each pretreatment year, as predictors. It turned out that matching quality of the covariates improved substantially at the cost of worse matching of birth rates. Therefore, with the current dataset, a trade-off must be made between birth rates and the covariates in terms of matching quality. Nevertheless, fertility effects of the two-child policy remained stable with changing sets of predictors.
} 
Table 2: Balancing properties

\begin{tabular}{|c|c|c|c|}
\hline & Yicheng & $\begin{array}{l}\text { Synthetic } \\
\text { Yicheng }\end{array}$ & $\begin{array}{l}\text { Average of } \\
\text { control counties }\end{array}$ \\
\hline$\%$ Female, 0-14 (city level) & 17.05 & 15.02 & 16.12 \\
\hline$\%$ Male, $0-14$ (city level) & 17.98 & 16.34 & 17.43 \\
\hline$\%$ Female, $15-59$ (city level) & 28.32 & 29.15 & 27.90 \\
\hline$\%$ Male, 15-59 (city level) & 29.60 & 33.16 & 30.73 \\
\hline$\%$ Female, 60+ (city level) & 3.58 & 3.08 & 3.84 \\
\hline$\%$ Female (county level) & 48.60 & 42.79 & 47.16 \\
\hline Deaths per 1,000 people & 7.28 & 5.28 & 7.12 \\
\hline Hospitals per 1,000 people & 0.07 & 0.22 & 0.18 \\
\hline$\%$ Agricultural population & 92.98 & 56.26 & 84.48 \\
\hline Log of GDP per capita & 5.24 & 6.17 & 5.86 \\
\hline Log of rural personal income & 4.38 & 4.54 & 4.56 \\
\hline Elementary schools per 1,000 people & 2.24 & 1.78 & 1.75 \\
\hline Middle schools per 1,000 people & 0.40 & 0.41 & 0.44 \\
\hline Births per 1,000 people (1972) & 18.98 & 20.22 & 29.70 \\
\hline Births per 1,000 people (1973) & 18.23 & 18.28 & 27.40 \\
\hline Births per 1,000 people (1974) & 17.73 & 17.85 & 26.39 \\
\hline Births per 1,000 people (1975) & 17.42 & 16.41 & 23.67 \\
\hline Births per 1,000 people (1976) & 15.35 & 15.51 & 19.76 \\
\hline Births per 1,000 people (1977) & 14.21 & 14.36 & 17.58 \\
\hline Births per 1,000 people (1978) & 14.05 & 13.34 & 15.91 \\
\hline Births per 1,000 people (1979) & 13.65 & 12.82 & 15.99 \\
\hline Births per 1,000 people (1980) & 12.16 & 12.78 & 16.16 \\
\hline Births per 1,000 people (1981) & 11.64 & 12.26 & 17.01 \\
\hline Births per 1,000 people (1982) & 12.10 & 13.84 & 18.78 \\
\hline Births per 1,000 people (1983) & 10.93 & 11.23 & 15.23 \\
\hline Births per 1,000 people (1984) & 10.96 & 11.26 & 14.42 \\
\hline
\end{tabular}

Data source: Please refer to Section 4.

One possible reason for the inverted $U$ patterns of both series may have been a change in policy enforcement. In Yicheng, as mentioned in previous sections, a large portion of second births occurring before the mid-1990s were unpermitted, which may have resulted in a fertility boom in the first decade of the two-child policy. After the mid-1990s, unpermitted second births became much less common due to stricter enforcement, which likely explains the downward trend in the county's fertility rate in 
the 2000s. In rural areas, the Chinese central government relaxed the OCP to a one-andhalf-child policy during the mid-1980s. However, because this policy relaxation was not clearly understood, a number of local authorities and residents believed that two children were generally allowed (Yang 2004). This may explain the rise in fertility rates in synthetic Yicheng, as well as the similarity in birth rates between Yicheng and its synthetic counterpart in the late 1980s. Shanxi legislated family planning in 1989, after which policy enforcement grew stricter (Yang 2004). This may have contributed to the fertility decline in the 2000s. In other words, the birth rate differences in 2000 and 2010 may better reflect the differences between the actual OCP and the pilot two-child policy. ${ }^{23}$ The 2000 and 2010 differences in CBR between Yicheng and synthetic Yicheng were 2.35 and 1.97 , respectively, indicating that the two-child policy would result in approximately two more births per 1,000 people each year compared with the OCP. Therefore, the long-term effects of this policy change were fairly small.

However, the estimated magnitude of this effect may be somewhat conservative in the short term for two reasons. On the one hand, because of the loose enforcement of the OCP in the synthetic Yicheng in the late 1980s, we could not precisely estimate the short-run effect of the two-child policy in its first five years. On the other hand, the fertility shock due to the two-child policy may not be as strong as today because two children were allowed prior to 1980 .

Given these two concerns, we re-estimated the potential impact of the two-child policy for the first few years under the following two assumptions: First, we assumed that the other counties in Shanxi maintained CBR levels as low as those in 1984; and second, we assumed that the CBR in Yicheng reached its peak, not as recorded in 1990, but immediately following the implementation of the two-child policy. These two assumptions allowed us to address the first concern raised above by re-estimating the upper bound of the CBR variation between Yicheng and its synthetic counterpart in the short term to 4.6 births per 1,000 people. Again, this is a rather limited number. Unfortunately, we did not have an effective method for addressing the second concern. However, as fertility declines with cohort, the responses to the relaxation of the twochild policy were also likely to decline with cohort. That said, the fertility peak in Yicheng immediately after 1985 may not have differed greatly from what might occur today after implementation of the nationwide two-child policy.

${ }^{23}$ Another reason for the rise in fertility rates in the late 1980 s has to do with the baby boomers reaching childbearing age in the late 1980s (Liang 2014a). 
Figure 4: Crude birth rate in Yicheng and 'synthetic Yicheng'

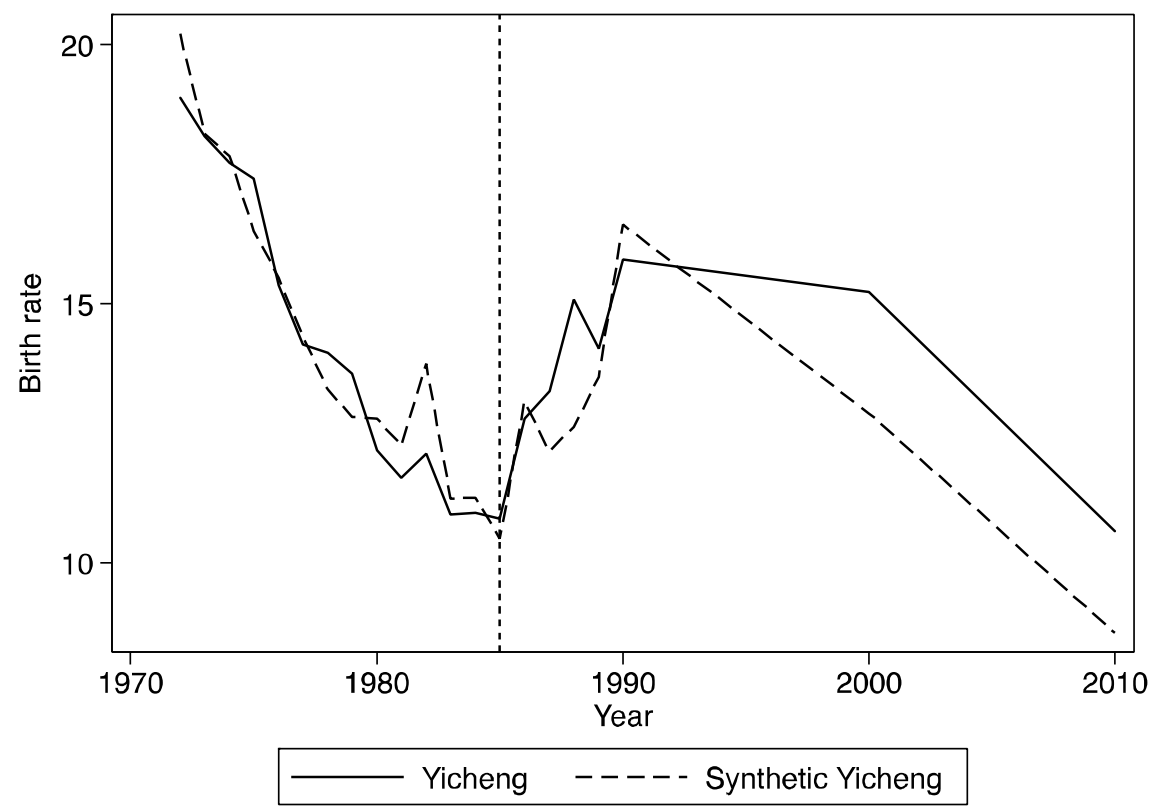

As mentioned in Abadie, Diamond, and Hainmueller (2010), our results could have been driven entirely by chance. Therefore, we ran placebo tests to determine the significance of our estimates. In Figure 5a, the dark line represents the variation in CBR between Yicheng and synthetic Yicheng over the years. We assumed each of the control units was the treated county, determined the variation in CBR between the county and its synthetic counterpart, and plotted the variation with a light line in Figure 5a. As a number of control units were poorly matched with their synthetic counterparts before the treatment, Figure $5 \mathrm{~b}$ illustrates only those counties with pretreatment MSPEs (mean squared prediction error, the average of the squared discrepancies between CBR in a county and its synthetic counterpart during the period 1972-1984) were not greater than that of Yicheng, reducing the number of counties from 115 to 62 . If the variations in Yicheng's CBR and synthetic Yicheng's CBR were mainly driven by the two-child policy rather than by chance, the dark line should remain above most, if not all, of the light lines. 


\section{Figure 5: Placebo tests}

a) All 115 counties

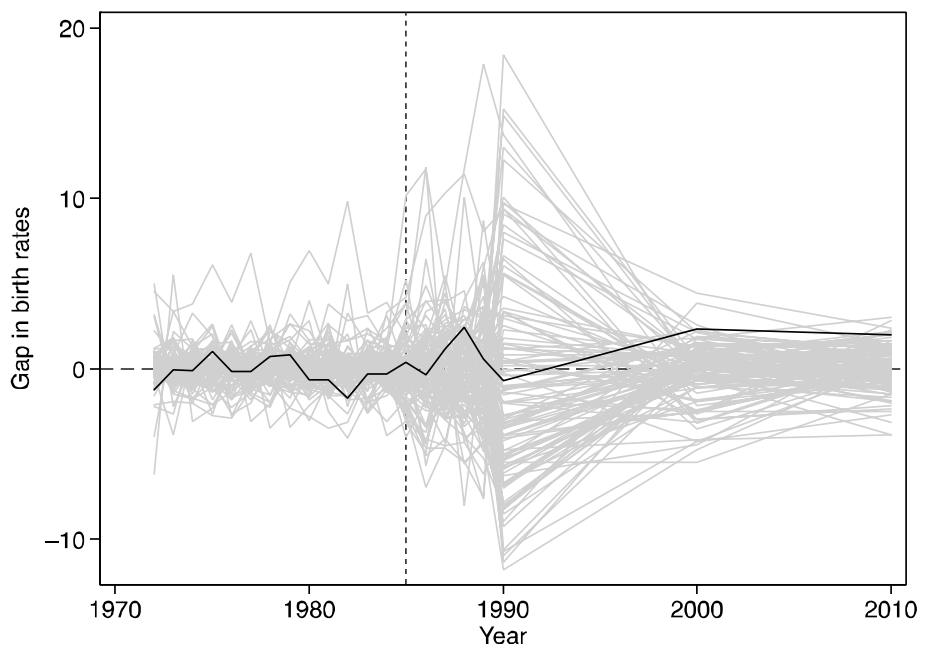

b) Yicheng and 61 counties with smaller pretreatment MSPE than Yicheng

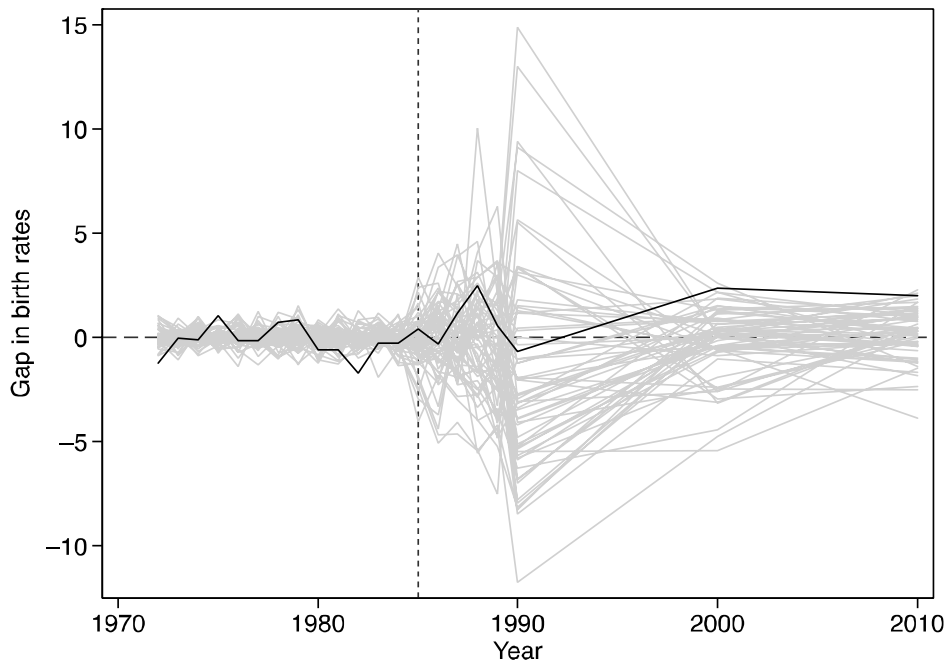


In both Figure 5 graphs, the dark Yicheng line does not stand out above all the light lines. However, in 2000 and 2010, the Yicheng values were clearly greater than those for most of the other counties. Table 3 also shows the percentiles for each year's CBR variation after the treatment. According to Table 3, Yicheng's policy effects were not significant between 1985 and 1990. However, in 2000 and 2010, the effects were significant at the level of $5 \%$ or $10 \%$.

Table 3: Percentage of counties having smaller treatment effects than Yicheng

\begin{tabular}{lll}
\hline Year & Full sample & Restricted sample \\
\hline 1985 & 69.6 & 74.2 \\
1986 & 43.5 & 38.7 \\
1987 & 68.7 & 74.2 \\
1988 & 89.6 & 85.5 \\
1989 & 61.7 & 61.3 \\
1990 & 58.3 & 62.9 \\
2000 & 96.5 & 96.8 \\
2010 & 92.2 & 95.2 \\
\# counties & 115 & 62 \\
\hline
\end{tabular}

Another concern that may have contaminated our estimates was cross-county migration. If the welcome policy in Yicheng attracted more migrants interested in having more children, the estimated effects might have been biased. However, this was an unlikely concern for two reasons. First, according to the 1990 census sample, the fraction of migrants in Yicheng did not increase from 1985 to 1990, the first five years of the two-child policy, and it remained below $2 \%$ in later years. Second, even though the estimated effects might have been biased, the true effects may be even smaller than estimated, which would not alter the conclusion that the two-child policy has a limited impact on fertility rates. As the fraction of migrants in Yicheng has been low, it is reasonable to argue that the attitudes and preferences regarding fertility of people in Yicheng were unlikely to be influenced by those in surrounding counties with strict one-child policies. In other words, the diffusion effect may be too small to substantially underestimate the effects of Yicheng's two-child policy on fertility.

Concerns over underreported births in China's datasets have been noticed by demographers (e.g., Zhang and Zhao 2006). If fertility rates suffered from a larger underestimation in regions with a harsher family planning policy, our estimated effects 
of the two-child policy on birth rates would be overrated. However, it is unlikely to change the conclusion that the two-child policy has a limited effect on birth rates.

\section{Robustness checks}

The synthetic control approach required that there be similarities between Yicheng and its donor pool of control counties. Constructing the synthetic Yicheng by only using counties that closely surround Yicheng might have made the assumption more robust. Unfortunately, neither the counties in Linfen prefecture, nor the counties in the neighbouring four prefectures of southern Shanxi, formed a well-matched synthetic Yicheng. By further including the counties in the four prefectures of central Shanxi, we were able to develop a synthetic Yicheng that fit well with the real-life case study, and the policy effects were similar to those from all of the counties in Shanxi province (Figure 6a). Moreover, the robustness check allowed us to confirm that if the county with the largest weight, Kuangqu in Datong prefecture, was excluded, the results would remain stable.

The effectiveness of the synthetic control method must also be evaluated over a sufficiently long pretreatment period. ${ }^{24}$ The results presented in the previous sections were based on matching specific characteristics in Yicheng with those from a donor pool of 114 counties for 1972-1984. For 1970-1984, data was available for Yicheng and 108 other counties. Figure $6 \mathrm{~b}$ shows the results from the comparison between Yicheng and the donor pool of 108 counties for 1970-1984. The extent of the similarities declined but was still acceptable. ${ }^{25}$ It turned out that the results remained robust if the pretreatment period is extended by two years. Unfortunately, further extension of the pretreatment period did not yield well-matched results. Compared with the literature, the pretreatment period used in our study was sufficiently long. ${ }^{26}$

\footnotetext{
${ }^{24}$ Abadie, Diamond, and Hainmueller (2010) indicated that the number of pretreatment periods should be large enough compared with the scale of the error term in the factor model. As the scale of the error term is immeasurable, there was no clear criterion for determining a proper length for the pretreatment period.

${ }^{25}$ The MSPE of the matching from 1970-1984 is about four times the MSPE of the matching from 19721984.

${ }^{26}$ In the classic example of Abadie, Diamond, and Hainmueller (2010), the pretreatment period is 19 years, similar to our best analysis, 15 years.
} 
Qin \& Wang: What have we learned from the 30-year two-child policy experiment in Yicheng, China?

Figure 6: Robustness checks

a) Using a small donor pool surrounding Yicheng

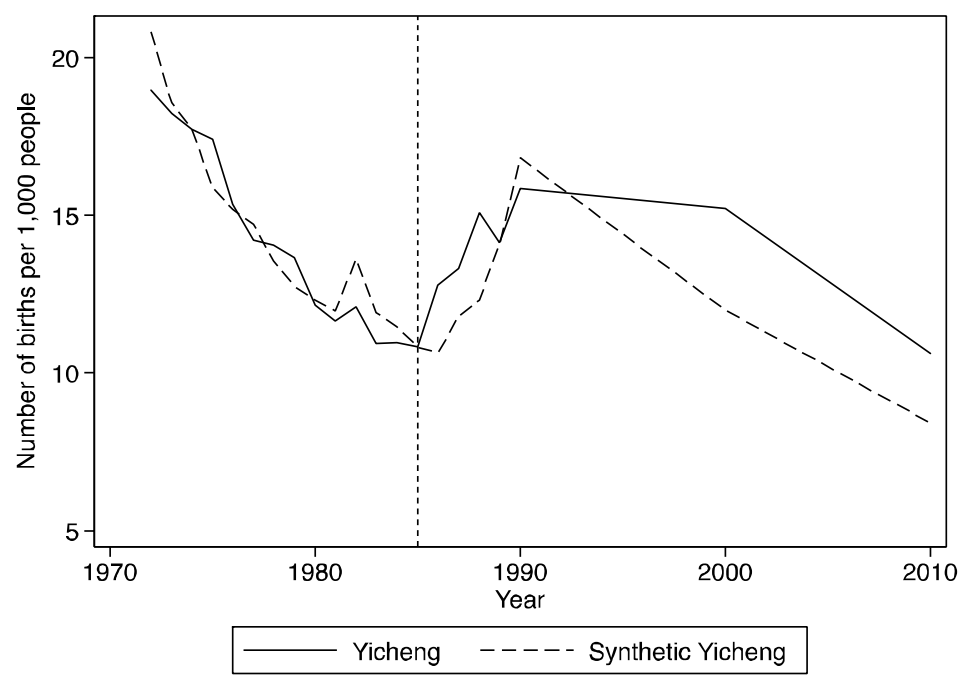

b) Extending the pretreatment period to 1970

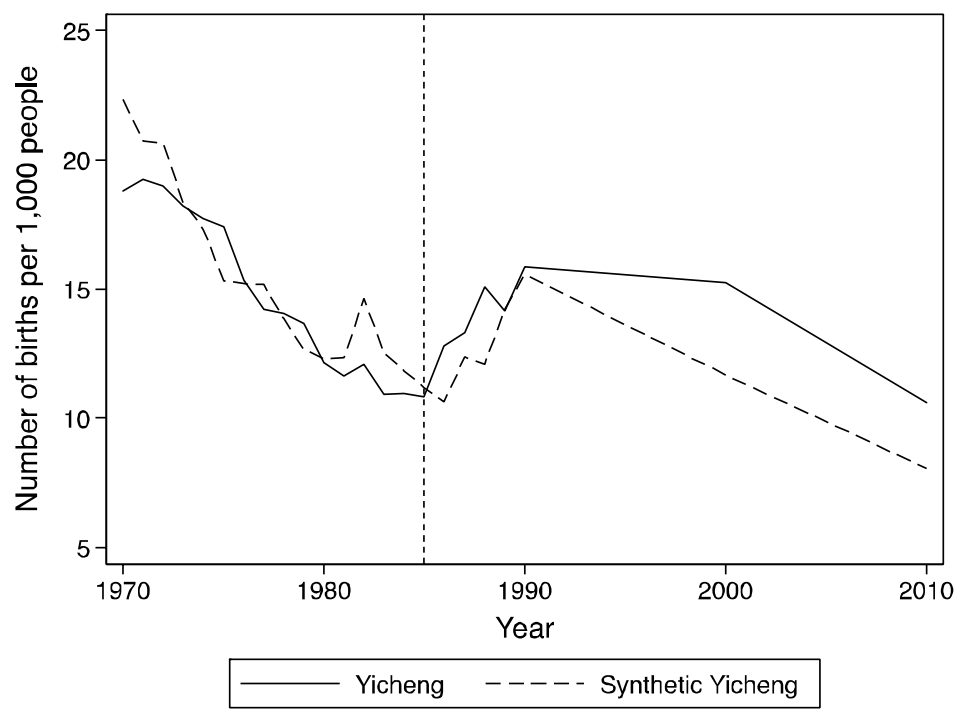


Figure 6: (Continued)

c) Using number of births per 1,000 females as outcome

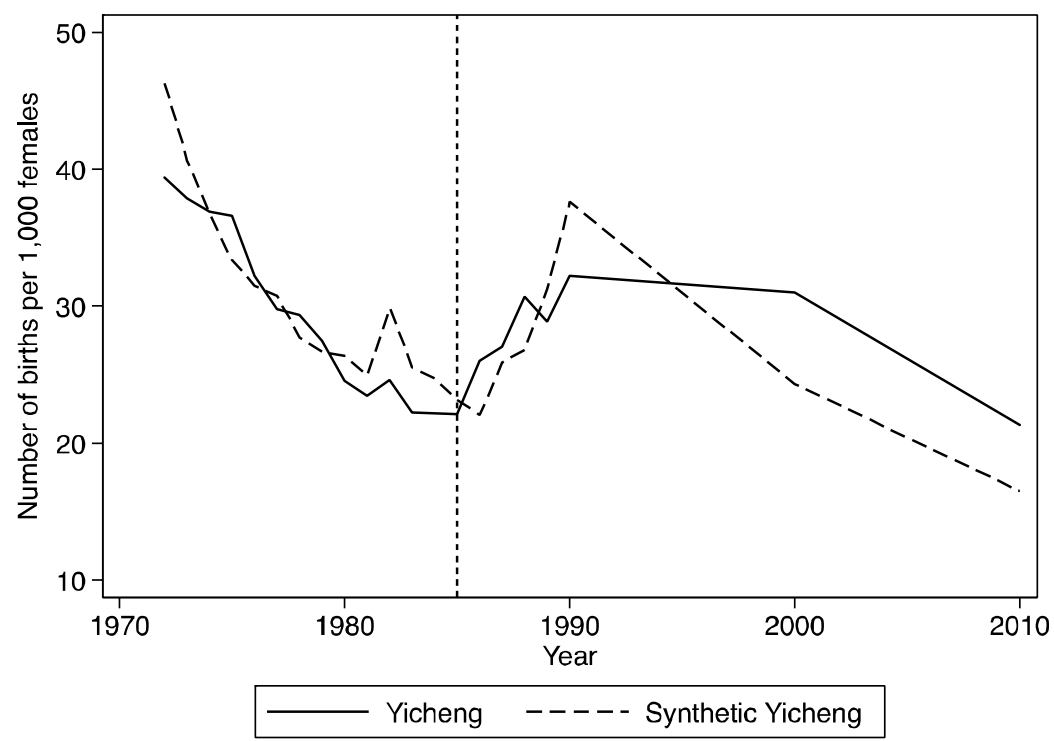

Despite the control of age and gender structure in model specifications, using CBR as the outcome may still result in biases caused by age and gender-specific variations. An alternative measure of birth rate is the general fertility rate, the number of births per 1,000 women during childbearing age. Unfortunately, the number of women of fertile ages was available only for the years $1982,1990,2000$, and 2010, and the variable in 1982 and 1990 was at the level of the prefecture city, not the level of county. Instead, we constructed the number of births per 1,000 females for each county and each year as an alternative outcome, and attempted to remove some gender-specific biases and applied the same procedure of optimization as in Section 6. Figure 6c shows trends of the new measure of birth rates for Yicheng and synthetic Yicheng. The pretreatment matching is acceptable, and fertility effects of the two-child policy in the 1990s and thereafter remained stable.

Other than the aggregate data collected by local authorities, census samples may also be used to assess the effects of a two-child policy on fertility rates. Despite 
concerns about the quality of data, ${ }^{27}$ we failed to find evidence of strong policy effects on fertility levels using the census samples, which was consistent with the results of the aggregate data obtained from local authorities.

\section{Conclusion}

In this paper, we studied the two-child policy experiment implemented in Yicheng beginning in 1985 . We adopted a synthetic control approach, which allowed us to conduct a counterfactual analysis by constructing a 'synthetic Yicheng' that mirrored the birth rate in Yicheng prior to the policy experiment. The estimated magnitude of the two-child policy's impact on the birth rate in our paper was lower than estimates predicted by influential demographers, such as Zhai, Zhang, and Jin (2014). They concluded that the relaxation of the OCP would likely to lead to an additional 97 million newborns in four years, equivalent to an approximate increase of 18 births per 1,000 people every year. Such population growth would slow the pace of aging in China. In contrast, our analysis suggests that replacing the OCP with a two-child policy will have a very limited impact on population growth, both in the short term and the long term, in rural areas such as Yicheng. Thus, it will have a very limited effect on the age distribution in those areas.

The synthetic control approach is more advantageous than potentially competing methods, such as DID and traditional matching, in certain respects. DID requires the assumption of parallel pretreatment trends of outcome, while the synthetic control approach may be applicable in the absence of the assumption. Traditional matching methods, such as propensity score matching, essentially assume selection on observables. Under certain conditions, the synthetic control approach could fulfil matching of unobservables (Abadie, Diamond, and Hainmueller 2010). Moreover, the synthetic control approach is suitable with as little as a single treatment unit, while DID and traditional matching with such a small number of treatment units would generate inaccurate estimation and inference.

Commensurate with its strength, the synthetic control approach has strict requirements on the quality of data. The data should contain sufficiently long pretreatment periods for outcome, and a donor pool of units that are similar enough to

\footnotetext{
${ }^{27}$ We constructed a series of CBR for Yicheng and other counties in Shanxi province using the $1 \%$ sample of the 1990 census, the $0.1 \%$ sample of the 2000 census, and the compiled statistics of the 2010 census. Sample sizes are small for each county and year in the samples of the 1990 and 2000 censuses, so that sampling errors would make inferences rather inaccurate. In addition, CBR before 1990 and in 1990-2000 were inferred from the population structure in the 1990 and 2000 census samples, respectively, on the premise of zero mortality rates over time, which may be substantially different from reality.
} 
the treated unit. Failure to satisfy these conditions could lead to biased estimates. Unlike other approaches, there are few methods for testing the assumptions needed by the synthetic control approach. We tried our best to make the data fit the requirements, and results remained stable after we extended the pretreatment period or selected a subset of the donor pool which was more similar to Yicheng, the treated unit. In addition to the untestable assumptions, estimators from synthetic control approach do not have a derived distribution, so that inference is executed indirectly and imprecisely using a placebo test.

A caveat of this paper is that the estimated impact of the two-child policy presented here may at best be generalized in rural arears similar to Yicheng, and cannot be generalized to other regions of China. This is due to the limitation of the synthetic control method since the estimated policy impact is specific to each treated unit. It is unfortunate that there were no other cases with persistent two-child policy that allowed us to examine the treatment effects and compare them with Yicheng's case. At the very least, the 30-year experiment in Yicheng suggests that it is not too early, but may be too late, to alter the aging trend using a two-child policy in such areas. ${ }^{28}$ Another limitation worth noting is that we only had the 2000 and 2010 data to estimate the long-term impact of the two-child policy. It would be beneficial if a greater number of years of data could be used for future research in order to depict a clearer pattern of the longterm effects.

\section{Acknowledgements}

This paper is indebted to the helpful comments of participants who attended the author's presentations at the 2015 CIER-IZA Annual Workshop on Research in Labor Economics, the 2015 International Symposium on Contemporary Labor Economics, the 2016 Annual Conference of the Population Association of America, as well as the seminars at the National School of Development, Peking University, and the Research Institute of Economics and Management, Southwestern University of Finance and Economics. We appreciate Associate Editor Jakub Bijak and two anonymous reviewers for their insightful suggestions, and Siyu Lu for excellent research assistance. Yu Qin acknowledges financial support from the Ministry in Singapore (grant no. R-297-000129-133). Fei Wang acknowledges financial support from the National Natural Science Foundation of China (grant no. 71703160).

\footnotetext{
${ }^{28}$ Simulations by Wang, Zhao, and Zhao (2017) have shown that the aging trend cannot be altered even if the birth quota is completely removed.
} 
Qin \& Wang: What have we learned from the 30-year two-child policy experiment in Yicheng, China?

\section{References}

Abadie, A., Diamond, A., and Hainmueller, J. (2010). Synthetic control methods for comparative case studies: Estimating the effect of California's tobacco control program. Journal of the American Statistical Association 105(490): 493-505. doi:10.1198/jasa.2009.ap08746.

Abadie, A., Diamond, A., and Hainmueller, J. (2015). Comparative politics and the synthetic control method. American Journal of Political Science 59(2): 495-510. doi:10.1111/ajps.12116.

Abadie, A. and Gardeazabal, J. (2003). The economic costs of conflict: A case study of the Basque Country. The American Economic Review 93(1): 113-132. doi: $10.1257 / 000282803321455188$.

Adsera, A. (2006). An economic analysis of the gap between desired and actual fertility: The case of Spain. Review of Economics of the Household 4(1): 75-95. doi:10.1007/s11150-005-6698-y.

Arnold, F. and Liu, Z. (1986). Sex preference, fertility, and family planning in China. Population and Development Review 12(2): 221-246. doi:10.2307/197 3109 .

Cameron, A.C. and Miller, D.L. (2015). A practitioner's guide to cluster-robust inference. Journal of Human Resources 50(2): 317-372. doi:10.3368/jhr.50.2. 317.

Easterlin, R.A. (1978). What will 1984 be like? Socioeconomic implications of recent twists in age structure. Demography 15(4): 397-432. doi:10.2307/2061197.

Easterlin, R.A. and Crimmins, E.M. (1985). The fertility revolution: A supply-demand analysis. Chicago and London: University of Chicago Press.

Fernández, R. and Fogli, A. (2006). Fertility: The role of culture and family experience. Journal of the European Economic Association 4(2-3): 552-561. doi:10.1162/jeea.2006.4.2-3.552.

Frisch, R.E. (1982). Fertility and population regulation: Demographic implications of the biological determinants of female fecundity. Social Biology 29(1-2): 187192. doi:10.1080/19485565.1982.9988489.

Gu, B. and Wang, F. (2009). An experiment of eight million people. Beijing: Social Sciences Academic Press. 
Lesthaeghe, R. and Vanderhoeft, C. (2001). Ready, willing and able: A conceptualization of transitions to new behavioural forms. In: Casterline, J.B. (ed.). Diffusion processes and fertility transition: Selected perspectives. Washington, D.C.: National Academy Press: 240-264.

Liang, Z. (2014a). Studies on China's fertility policies. Taiyuan: Shanxi People's Press.

Liang, Z. (2014b). China population problem. Beijing: China Development Press.

McDonald, P. (2006). Low fertility and the state: The efficacy of policy. Population and Development Review 32(3): 485-510. doi:10.1111/j.1728-4457.2006.0013 4.x.

United Nations (2013). World Population Policies 2013. New York: United Nations Publication.

Wang, F. (2014). Essays on family planning policies. [PhD thesis]. Los Angeles: University of Southern California, Dana and David Dornsife College of Letters, Arts and Sciences, Department of Economics.

Wang, F., Zhao, L., and Zhao, Z. (2017). China's family planning policies and their labor market consequences. Journal of Population Economics 30(1): 31-68. doi:10.1007/s00148-016-0613-0.

Wang, G., Hu, Y., and Zhang, L. (2013). Adjustment of family planning policy in China. Beijing: Social Sciences Academic Press.

Wei, Y. and Zhang, L. (2014). Re-examination of the Yicheng Two-Child Program. China Journal 72: 98-120. doi:10.1086/677059.

Wood, J.W. (1989). Fecundity and natural fertility in humans. Oxford Reviews of Reproductive Biology 11: 61-109.

Wu, Y. (2014). Preliminary analysis of a second-child experiment on 'later marry, later birth and longer interval' in Yicheng county. Population Journal 4: 103-112.

Yang, F. (2004). Historical research on family planning of contemporary China. [PhD thesis]. Hangzhou: Zhejiang University, School of Humanities.

Zhai, Z., Zhang, X., and Jin, Y. (2014). Demographic consequences of an immediate transition to a universal two-child policy. Population Research 38(2): 3-17.

Zhang, G. and Zhao, Z. (2006). Reexamining China's fertility puzzle: Data collection and quality over the last two decades. Population and Development Review 32(2): 293-321. doi:10.1111/j.1728-4457.2006.00119.x. 
Qin \& Wang: What have we learned from the 30-year two-child policy experiment in Yicheng, China?

Zhang, J. (1990). Socioeconomic determinants of fertility in China. Journal of Population Economics 3(2): 105-123. doi:10.1007/BF00187287. 